\title{
Lowering HIV fitness and replication rate by administration of lamivudine alone, in extensively resistant HIV-infected patients, as a "bridging" strategy towards optimized salvage regimens
}

\author{
Roberto Manfredi
}

From $16^{\text {th }}$ International Symposium on HIV and Emerging Infectious Diseases

Marseille, France. 24-26 March 2010

\section{Background}

HIV-infected patients harbouring a lamivudine-resistant virus, seem to take benefit from a continued lamivudine monotherapy, versus combined antiretroviral treatment (cART) interruption, since a reduced HIV replication is selected by the maintenance of lamivudine-related M184 mutation. The mid-term outcome of isolated lamivudine therapy in multidrug-resistant patients with very restricted therapeutic options, waiting for novel drug classes, is reported.

\section{Methods}

Six patients aged 23-49 years (4 males and 2 females, one of them with perinatal infection), with HIV disease treated since $13.8 \pm 6.2$ years with $10.3 \pm 4.7$ therapeutic lines, experienced repeated virological-immunological failures due to an extensive HIV genotype resistance, which finally led to a complete 3-class resistance, and no residual therapeutic options, when excluding the use of a fusion/integrase/coreceptor inhibitors, without the possibility to optimize the therapeutic background. A concurrent toxicity was also present:combined lipodistrophy syndrome, dyslipidemia, and insulin resistance (3,2, and one patients, respectively).

\section{Results}

At the time of lamivudine monotherapy initiation, the median viremia was 36,000 HIV-RNA copies $/ \mathrm{mL}$, while the median CD4+ count was 344 cells $/ \mu \mathrm{L}$. Despite a previous diagnosis of AIDS in 4/6 patients, at the time of therapeutic switch the clinical situation was stable. During the monthly follow-up with lamivudine monotherapy,

Correspondence: Roberto.manfredi@unibo.it

Infectious Diseases, University of Bologna, S. Orsola Hospital, Bologna, Italy ranging from 8 to 24 months (mean $9.9 \pm 5.2$ ) months, no HIV-associated signs-symptoms occurred, previous cARTassociated laboratory toxicity significantly ameliorated, and no significant differences were found as to virologicalimmunological markers of HIV disease. A fluctuating viremia was noticed in all cases, with a median value at the end of follow-up of 44,000 HIV-RNA copies/mL, while no significant loss of CD4+ count occurred (median final levels:322 cells $/ \mu \mathrm{L}$ ). Two-four nucleos(t)ide mutations, and 2-5 protease mutations were deselected during the followup, but the M184 mutation remained. All these patients were allowed to re-introduce a cART with novel drug classes, according to the availability of an optimized therapeutic background in the subsequent months.

\section{Discussion}

When extensive HIV resistance does not leave therapeutic options, lamivudine monotherapy performed with a strict monitoring in clinically stable patients with no compromised virological-immunological figures, is a potentially safe choice. Waiting for the novel cART associations, the exploitment of lamivudine resistance on HIV replication-fitness represents an ultimate therapeutic approach to these difficult-to-manage subjects.

Published: 11 May 2010

doi:10.1186/1742-4690-7-S1-P58

Cite this article as: Manfredi: Lowering HIV fitness and replication rate by administration of lamivudine alone, in extensively resistant HIVinfected patients, as a "bridging" strategy towards optimized salvage regimens. Retrovirology 2010 7(Suppl 1):P58. 